\title{
Diagnostic value of FIB-4 for liver fibrosis in patients with hepatitis B: a meta-analysis of diagnostic test
}

\author{
Zhi Yinn ${ }^{1,2}$, Jin Zou ${ }^{3}$, Qiongxuan Li $^{1}$, Lizhang Chen ${ }^{1}$ \\ ${ }^{1}$ Department of Epidemiology and Health Statistics, Xiangya School of Public Health, Central South University, Changsha, \\ Hunan Province 410078, China \\ ${ }^{2}$ Division of Recruitment and Employment, University of South China, Hengyang, Hunan Province 421001, China \\ ${ }^{3}$ Department of Cardiology, The First Affiliated Hospital of University of South China, Hengyang, Hunan Province 421001, \\ China \\ Correspondence to: Lizhang Chen, email: liche4005@126.com
}

Keywords: liver fibrosis, hepatitis B, FIB-4, meta-analysis

Received: November 14, $2016 \quad$ Accepted: December 13, $2016 \quad$ Published: January 02, 2017

\section{ABSTRACT}

This study is aimed at evaluating the diagnostic value of FIB-4 for liver fibrosis in patients with hepatitis B through a meta-analysis of diagnostic test. We conducted a comprehensive search in the Pubmed, Embase, Web of Science, and Chinese National Knowledge Infrastructure before October 31, 2016. Stata 14.0 software was used for calculation and statistical analyses. We used the sensitivity, specificity, positive and negative likelihood ratio (PLR, NLR), diagnostic odds ratio (DOR) and $95 \%$ confidence intervals (CIs) to evaluate the diagnostic value of FIB-4 for liver fibrosis in patients with hepatitis B. Twenty-six studies were included in the final analyses, with a total of $\mathbf{8 2 7 4}$ individuals. The pooled parameters are calculated from all studies: sensitivity of 0.69 (95\%CI:0.63-0.75), specificity of 0.81 (95\%CI: $0.73-0.87)$, PLR of 3.63 (95\%CI: 2.66-4.94), NLR of 0.38 (95\%CI:0.32-0.44), DOR of 9.57 (95\%CI: 6.67-13.74), and area under the curve (AUC) of 0.80 (95\%CI: $0.76-0.83$ ). We also conducted subgroup based on the range of cut-off values. Results from subgroup analysis showed that cut-off was the source of heterogeneity in the present study. The sensitivity and specificity of cut-off $>2$ were 0.69 and 0.95 with the AUC of 0.90 (95\%CI: 0.87-0.92). The overall diagnostic value of FIB-4 is not very high for liver fibrosis in patients with hepatitis B. However, the diagnostic value is affected by the cut-off value. FIB-4 has relatively high diagnostic value for detecting liver fibrosis in patients with hepatitis B when the diagnostic threshold value is more than 2.0.

\section{INTRODUCTION}

It is reported that 24 billion populations have been exposed to hepatitis B virus (HBV). Three point five people are patients with chronic hepatitis B virus, and 75 million population died of hepatic failure, liver cirrhosis, liver cancer caused by HBV infection. Hepatitis B has become one of important public health issues [1]. Early detection and identification of liver fibrosis to prevent progression to cirrhosis is the goal of treatment in in patients with hepatitis B. In the past decades, liver biopsy has been considered as the gold standard for determining liver fibrosis. However, this invasive operation is related to several disadvantages including sampling error and some variations [2]. These application limitations result in developing dependable, non-invasive methods to detect the stage of fibrosis in patients with hepatitis B. Recently, research has focused on the development of noninvasive tests for the evaluation of liver fibrosis; serum-based tests have attracted the maximum attention One of the previous methods is Fibro test which was conducted in patients with hepatitis C. Subsequent models: aspartate aminotransferase to platelet ratio index (APRI), Forn's score, ELF-score, Hepascore Fibrometer have been studied worldwide [3]. The use of noninvasive indices such as the aspartate aminotransferase to platelet ratio index (APRI), the fibrosis index based on the four factors (FIB-4: the FIB-4 - a test derived from the Apricot database, which produces interesting results using the following formula: age (years) $\times$ AST $[\mathrm{U} / \mathrm{L}] /($ platelets $\left.\left[10^{9} / \mathrm{L}\right] \times(\mathrm{ALT}[\mathrm{U} / \mathrm{L}])^{1 / 2}\right)$ score [4], and the Forn's index 
has been suggested as a method to assess liver fibrosis in patients with chronic liver diseases [5].

The non-invasive diagnostic methods have become a new field. There are studies reported that the diagnostic accuracy of FIB-4 for liver fibrosis in patients with hepatitis B. However, the diagnostic ability from different study is obviously different, which may be affected by some limitations such as sampling error, inter-and intraobserver variations. Considering the limitation of single study, we try to conduct this meta-analysis based on more study samples and statistics, aiming to acquire the diagnostic efficiency of FIB-4 for liver fibrosis in patients with hepatitis B more accurately.

\section{RESULTS}

\section{Study selection and study characteristics}

The selection flow of literature search was presented in Figure 1. Our initial search obtained 15 records. 95 duplicates records were removed, and 262 records were excluded after reviewing titles, abstract and topic. 58 fulltext articles were assessed for eligibility. With a further work on reading full texts of 58 articles, we removed 20 records unrelated to diagnostic value, and 4 duplicates. Similarly, 8 articles were excluded for unable to provided sufficient data. Finally, 26 articles were entered into the final qualitative and quantitative analyses [3, 5-29].

In Table 1, we summarized the characteristics of the 26 included articles in the meta-analysis of FIB-4 for liver fibrosis. The publication year of 26 studies ranged from 2009 to 2015. The studies were from different countries all over the world, and included 8274 individuals. Of the 26 studies, 3 studies are prospective design and 23 are retrospective. Most of them are multicenter studies. The sample size varied from 52 to 2411. Among the 26 studies, 18 studies were conducted in Asian (China, India, Korea), 4 in Caucasian population, 3 in Central East, and 1 in African population. The four-fold table data was presented in Table 1.

\section{Quality assessment}

The quality score of each study was presented in Table 1. The score of each study was more than 11 points. All the included studies received moderately high scores from the QUADAS-2 quality assessments.

\section{Pooled diagnostic values}

We use the random effect model to pool the sensitivity and specificity because the $I^{2}$ values were more than $50 \%$. The pooled diagnostic values of FIB4 for detecting liver fibrosis in patients with hepatitis B were presented in Table 2. The overall pooled sensitivity and specificity were 0.69 (95\%CI:0.63-0.75,
Figure 2) and 0.81 (95\%CI: 0.73-0.87, Figure 3). The pooled PLR was 3.63 (95\%CI:2.66-4.94), NLR was 0.38 (95\%CI:0.32-0.44), and DOR was 9.57 (95\%CI: 6.6713.74). The overall SROC curve was shown in Figure 4, and AUC was 0.81 (95\%CI: 0.77-0.84). Fagan plot was shown in Figure 5. The prior probability was $20 \%$, and the post-test probability was $48 \%$ of LR-positive, and $9 \%$ of LR-negative. The diagnostic accuracy of FIB-4 for detecting liver fibrosis in patients with hepatitis B was not very high.

We subgroup analyses based on the range of cutoff values. Results from subgroup analysis showed that cut-off was the source of heterogeneity in the present study. The sensitivity and specificity of cut-off $>2$ were 0.69 (95\%CI: $0.60-0.84$ ) and 0.95 (95\%CI: $0.83-0.99$ ) with the AUC of 0.90 (95\%CI: 0.87-0.92). The pooled diagnostic values other two cut-off range was presented in Table 2.

\section{Sensitivity analysis and publication bias}

Sensitivity analysis was conducted by sequentially excluding some special studies (with small sample size, bigger cut-off value) [20,27]. The pooled results did not alter, indicating the results were stable. The Deek's plot shows there was no publication bias $(\mathrm{t}=1.670, P=0.107$, Figure 6).

\section{DISCUSSION}

With the increasing popularity of hepatitis B over the world, it is needed for effective and convenient diagnostic methods. In the past decades, the arise of non-invasive and new methods in detecting liver fibrosis had developed rapidly. Among all kinds of examination methods, more and more researchers paid attention on combined diagnostic index. FIB-4, one of the novel methods in detecting liver fibrosis, attracted more attention and was widely explored for its role in patients with hepatitis B [5].

In this meta-analysis, we found FIB-4 achieved the overall pooled sensitivity of 0.69 (95\%CI:0.630.75 ) and specificity 0.81 (95\%CI: $0.73-0.87)$, and AUC was 0.81 (95\%CI: $0.77-0.84)$. The results showed that the diagnostic accuracy may not be high enough as expected. However, FIB-4 still achieves almost same level diagnostic value with some invasive methods [21]. The DOR represents the value that combines sensitivity and specificity that ranges from 0 to infinity, with higher value meaning better diagnostic ability. Our results show the DOR was 9.57 (6.67-13.74), suggesting the overall pooled accuracy was not high. The pooled PLR of 3.63 means that patients with liver fibrosis have approximately a 3.63-fold higher chance of being FIB-4 positive compared with individuals without liver fibrosis. 
The pooled NLR was 0.38 , indicating that the probability of patients having liver fibrosis is 38\% if the FIB-4 was negative. According to the criteria, the accuracy is considered to be high when PLR $>10$ and NLR $<0.1$. The present result did arrive the standard, suggesting that FIB-4 had sub-optimal accuracy for clinical purpose [30]. However, FIB-4 has relatively high diagnostic value for detecting liver fibrosis in patients with hepatitis B when the diagnostic threshold value was more than 2.0.
This result indicates that FIB had higher accuracy for developed liver fibrosis in patients with hepatitis B. Considered the diagnostic ability of other non-invasive methods, the advantage of FIB-4 is not outstanding in severe liver fibrosis stage. Previous studies reported that the aspartate transaminase/blood platelet index (APRI) and Fibro were also potential non-invasive methods. Previous meta-analysis suggested that AUCs of APRI and Fibro for detecting liver fibrosis were 0.75 and 0.87

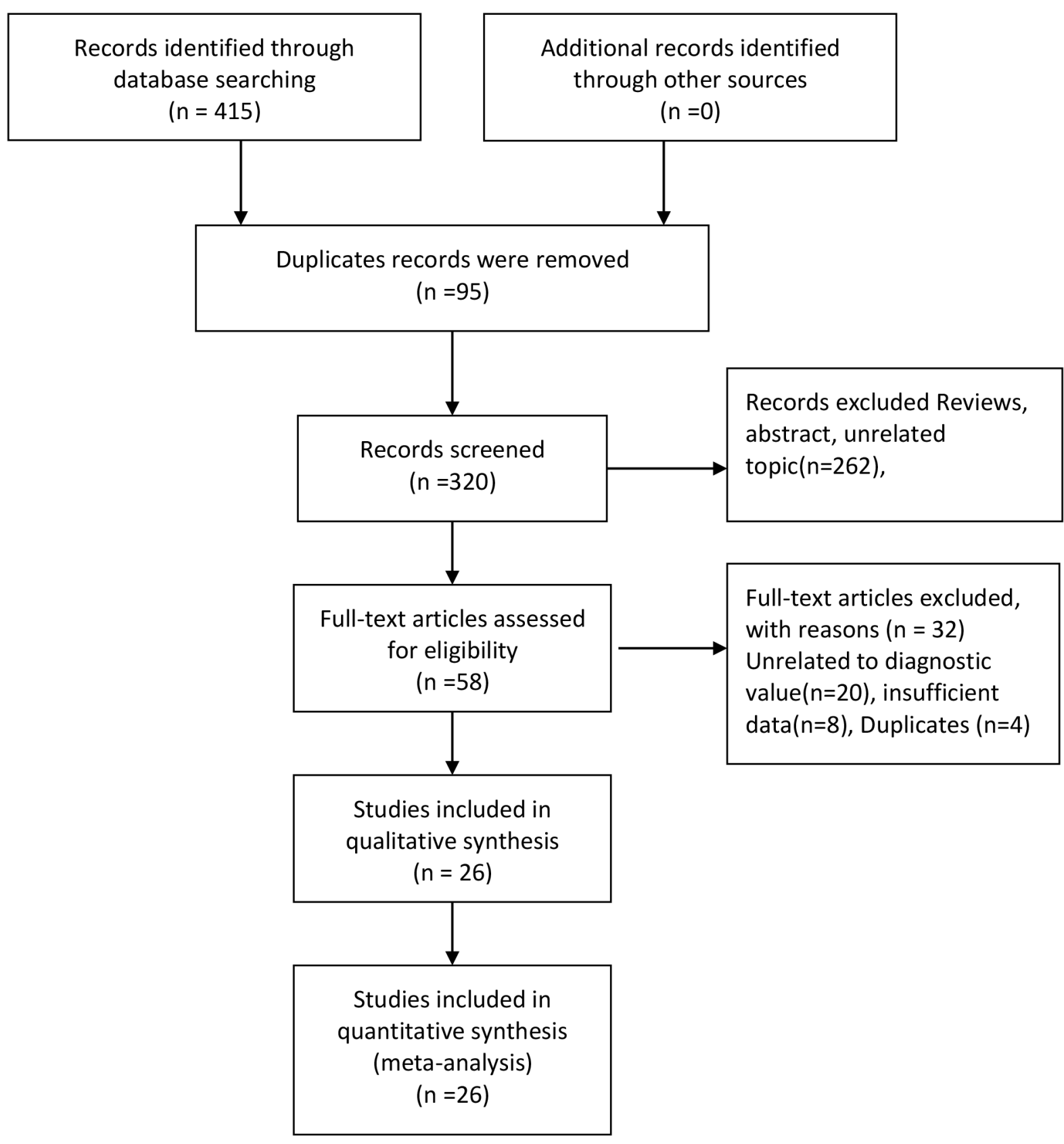

Figure 1: Flow diagram of studies selection process. 
Table 1: Characteristics of the included studies in the meta-analysis

\begin{tabular}{|c|c|c|c|c|c|c|c|c|c|c|c|c|c|c|}
\hline No. & Author & Year & Region & $\begin{array}{l}\text { Mean } \\
\operatorname{age}(y)\end{array}$ & $\begin{array}{l}\text { Sample } \\
\text { size }\end{array}$ & Study design & $\begin{array}{c}\text { Study } \\
\text { population }\end{array}$ & $\begin{array}{l}\text { Length } \\
\text { of tissue }\end{array}$ & Cut off & TP & FP & FN & TN & $\begin{array}{l}\text { Score of } \\
\text { Quality }\end{array}$ \\
\hline 1 & $\mathrm{Li}$ & 2013 & USA & 45 & 284 & Retrospective & Multicenter & - & 5.17 & 19 & 1 & 32 & 232 & 13 \\
\hline 2 & Koksal & 2015 & USA & 43 & 229 & Retrospective & Multicenter & - & 1.45 & 98 & 5 & 94 & 32 & 12 \\
\hline 3 & Erdogan & 2013 & Turkey & 41 & 221 & Retrospective & Single center & - & 1.02 & 53 & 63 & 15 & 90 & 12 \\
\hline 4 & Ucar & 2013 & Turkey & 45 & 73 & Retrospective & Single center & - & 1.09 & 29 & 12 & 12 & 20 & 13 \\
\hline 5 & Shrivastava & 2013 & India & 30 & 52 & Retrospective & Single center & $15-20$ & 2.50 & 2 & 2 & 4 & 44 & 14 \\
\hline 6 & Basar & 2013 & Turkey & 45 & 76 & Prospective & Single center & $>10$ & 1.09 & 37 & 6 & 14 & 19 & 14 \\
\hline 7 & Seto & 2011 & H.K. & 38 & 237 & Retrospective & Single center & $>15$ & 1.45 & 40 & 41 & 37 & 119 & 14 \\
\hline 8 & Sebastiani & 2011 & French & 47 & 2411 & Retrospective & Multicenter & - & 1.45 & 784 & 455 & 328 & 844 & 14 \\
\hline 9 & Kim & 2010 & Korea & 39 & 668 & Retrospective & Single center & $>15$ & 1.00 & 301 & 92 & 29 & 246 & 14 \\
\hline 10 & Bonnard & 2010 & Africa & 35 & 59 & Prospective & Single center & - & 0.80 & 30 & 7 & 11 & 11 & 12 \\
\hline 11 & Mallet & 2009 & French & 42 & 138 & Retrospective & Multicenter & 17.6 & 1.45 & 29 & 26 & 12 & 71 & 13 \\
\hline 12 & $\mathrm{Wu}$ & 2010 & China & 33 & 78 & Retrospective & Multicenter & $>15$ & 1.45 & 20 & 7 & 12 & 39 & 14 \\
\hline 13 & Liu & 2011 & China & 32 & 623 & Retrospective & Multicenter & $>15$ & 1.10 & 158 & 130 & 57 & 278 & 13 \\
\hline 14 & Zhu & 2011 & China & 37 & 175 & Retrospective & Multicenter & $>15$ & 1.70 & 57 & 15 & 22 & 81 & 12 \\
\hline 15 & $\mathrm{Wu}$ & 2012 & China & 33 & 482 & Retrospective & Multicenter & $>15$ & 1.57 & 189 & 66 & 81 & 146 & 13 \\
\hline 16 & Zhu & 2012 & China & 42 & 159 & Prospective & Single center & $>15$ & 4.90 & 91 & 10 & 13 & 45 & 12 \\
\hline 17 & Chen & 2013 & China & 40 & 148 & Retrospective & Single center & $>15$ & 1.45 & 27 & 29 & 13 & 79 & 13 \\
\hline 18 & Wang & 2013 & China & 34 & 231 & Retrospective & Single center & $>15$ & 1.45 & 37 & 24 & 31 & 139 & 11 \\
\hline 19 & Wang & 2013 & China & 37 & 149 & Retrospective & Multicenter & $>10$ & 1.45 & 60 & 21 & 29 & 39 & 12 \\
\hline 20 & Xun & 2013 & China & 31 & 197 & Prospective & Single center & $>15$ & 1.00 & 80 & 26 & 32 & 59 & 13 \\
\hline 21 & Zeng & 2013 & China & 36 & 198 & Retrospective & Single center & $15-20$ & 31.61 & 25 & 35 & 13 & 125 & 12 \\
\hline 22 & Liu & 2014 & China & 38 & 111 & Retrospective & Single center & 16.67 & 2.29 & 11 & 28 & 1 & 74 & 12 \\
\hline 23 & $\mathrm{Xu}$ & 2015 & China & 36 & 446 & Retrospective & Single center & $>16$ & 1.07 & 160 & 84 & 59 & 143 & 11 \\
\hline 24 & $\mathrm{Li}$ & 2013 & China & 45 & 284 & Retrospective & Multicenter & - & 5.17 & 19 & 1 & 32 & 232 & 11 \\
\hline 25 & $\mathrm{Ji}$ & 2011 & China & 36 & 313 & Retrospective & Single center & $>20$ & 2.96 & 44 & 19 & 6 & 244 & 11 \\
\hline 26 & $\mathrm{Li}$ & 2015 & China & 38 & 232 & Retrospective & Single center & - & 1.58 & 66 & 46 & 20 & 152 & 11 \\
\hline
\end{tabular}

$[31,32]$. The diagnostic ability of FIB-4 is prior to APRI but less than Fibro. The FIB-4 was firstly applied in the diagnostic of hepatitis $\mathrm{C}$. It is reported that the AUC of FIB-4 was 0.74 in patients with hepatitis C. The FIB-4 in hepatitis B was obviously higher than in the hepatitis $\mathrm{C}[33]$.

Our results found that the diagnostic of FIB-4 for liver fibrosis had many different kinds of cut-off values. It is pretty difficult for clinical practice. Therefore, this studies conducted subgroup analyses based the range of cut-off values. The diagnostic threshold value of FIB4 achieves the highest AUC when the cut-off was more than 2. The corresponding AUC was 0.90 (95\%CI: $0.87-$ 0.92 ) with the sensitivity of 0.69 and the specificity of
0.95, indicating the diagnostic ability within this interval is higher than other range. However, there are still some studies with so high cut-off values that they can't be pooled in the meta-analysis. Xu et al used the 223.7 as the cut-off value of liver fibrosis in the patients with hepatitis B, which can't be included in the final analyses. More study with strict design, larger sample size, and multicenter are required to test the accuracy of FIB-4 and identify the proper cut-off value range.

We strictly follow the PRISM guidelines to conduct the meta-analysis [34]. But, there are still some limitations in the present meta-analysis. First, although we have tried our best to search relevant studies, we may neglect some studies non-published 
Table 2: Summary estimated of diagnostic performance of FIB-4 for liver fibrosis

\begin{tabular}{lcccccc}
\hline Category & $\begin{array}{c}\text { SEN } \\
(\mathbf{9 5 \%} \mathbf{0})\end{array}$ & $\begin{array}{c}\text { SPE } \\
\mathbf{( 9 5 \% C I )}\end{array}$ & $\begin{array}{c}\text { PLR } \\
\mathbf{( 9 5 \% C I )}\end{array}$ & $\begin{array}{c}\text { NLR } \\
\mathbf{( 9 5 \% C I )}\end{array}$ & $\begin{array}{c}\text { DOR } \\
\mathbf{( 9 5 \% C I )}\end{array}$ & $\begin{array}{c}\text { AUC } \\
(\mathbf{9 5 \%} \% \mathbf{C I})\end{array}$ \\
\hline Overall & $0.69[0.63-0.75]$ & $0.81[0.73-0.87]$ & $3.63[2.66-4.94]$ & $0.38[0.32-0.44]$ & $9.57[6.67-13.74]$ & $0.80[0.76-0.83]$ \\
$0.8-1.1$ & $0.77[0.70-0.82]$ & $0.66[0.63-0.70]$ & $2.29[1.95-2.68]$ & $0.35[0.26-0.47]$ & $6.52[4.18-10.19]$ & $0.72[0.68-0.76]$ \\
$1.2-2.0$ & $0.65[0.60-0.70]$ & $0.76[0.711-0.81]$ & $2.72[2.28-3.24]$ & $0.46[0.41-0.52]$ & $5.88[4.59-7.55]$ & $0.76[0.72-0.79]$ \\
$>2$ & $0.69[0.60-0.84]$ & $0.95[0.83-0.99]$ & $12.89[4.47-37.18]$ & $0.33[0.19-0.57]$ & $39.17[16.13-95.13]$ & $0.90[0.87-0.92]$ \\
\hline
\end{tabular}

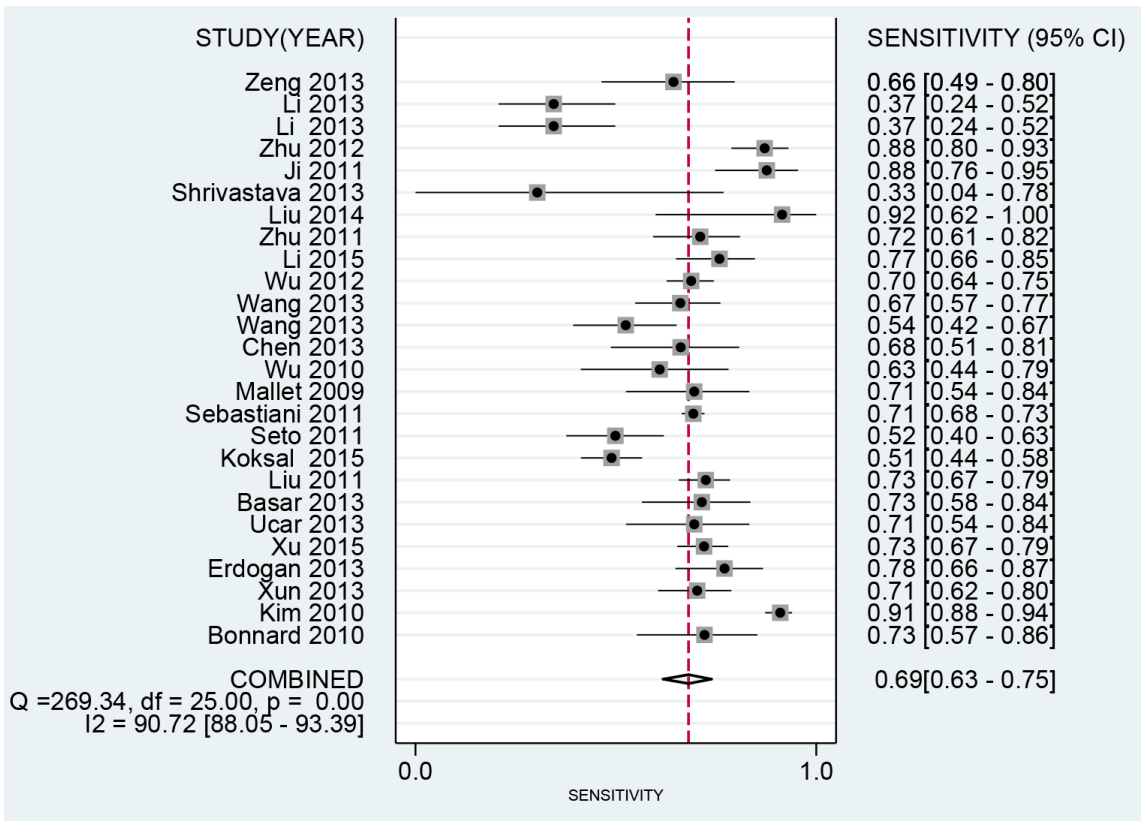

Figure 2: Forest plot of pooled sensitivity of FIB-4 for liver fibrosis in patients with hepatitis B.

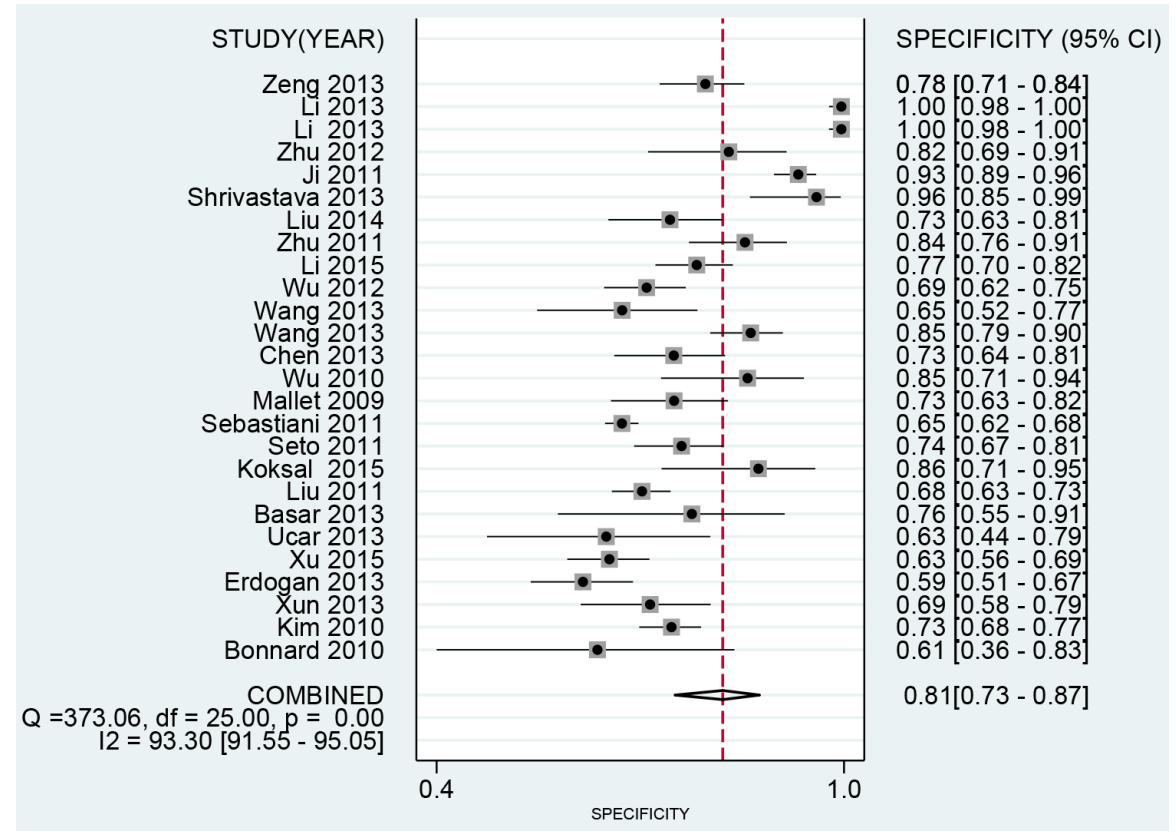

Figure 3: Forest plot of pooled specificity of FIB-4 for liver fibrosis in patients with hepatitis B. 


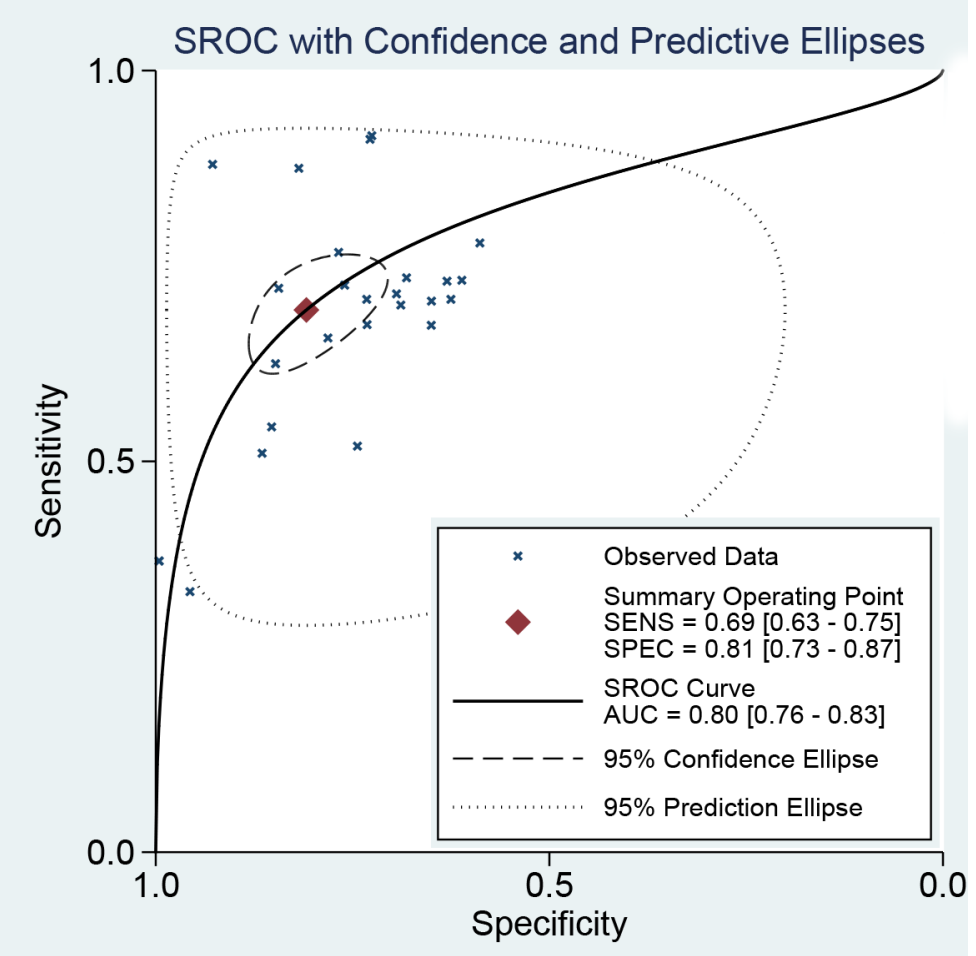

Figure 4: The SROC curve of FIB-4 for liver fibrosis in patients with hepatitis B.

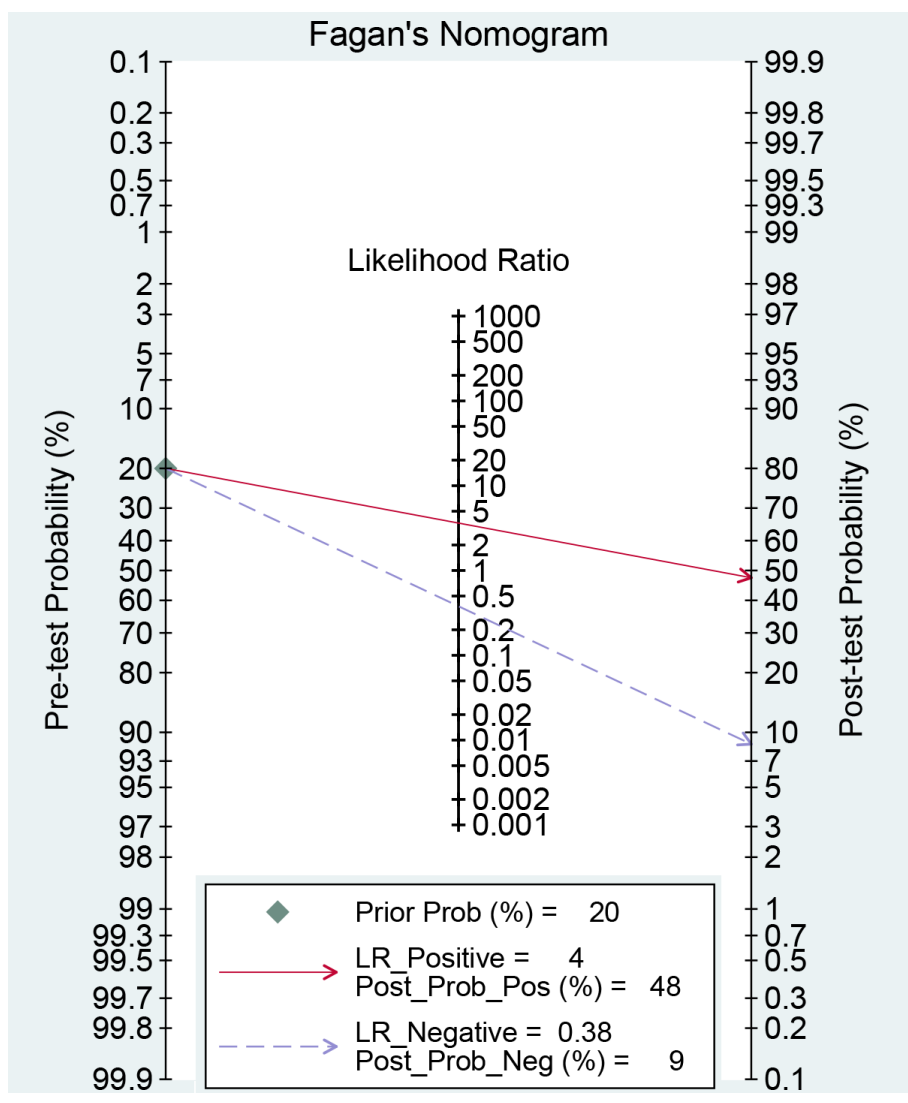

Figure 5: Fagan diagram evaluating the overall diagnostic value of FIB-4 for liver fibrosis in patients with hepatitis B. 
online. Second, there could exist some selection bias of population in the meta-analysis because the present studies only one African population, and most of studies are from Asian population. Third, the condition of study population included in the meta-analysis may have other diseases, which could overestimate or underestimate the diagnostic ability of FIB-4 for liver fibrosis. Four, the overall heterogeneity was high $\left(I^{2}: 88.0 \%-95 \% .0\right)$, we did not found the source of heterogeneity by subgroup. Finally, we did not consider the relation between sample quality and different cut-off values. Although our results presented that FIB-4 has relatively high diagnostic value for detecting liver fibrosis in patients with hepatitis $\mathrm{B}$ when the diagnostic threshold value was more than 2.0, the diagnostic ability of FIB-4 would be different in different progression of liver fibrosis such as liver cirrhosis. For different stage of liver fibrosis, it requires more studies and analyses to confirm the diagnostic value of FIB-4 in specific stage fibrosis.

\section{CONCLUSIONS}

In conclusions, our analysis showed that the overall diagnostic value of FIB-4 may not be very high and have sub-optimal accuracy for liver fibrosis in patients with hepatitis B. However, the diagnostic value is affected by the cut-off value. FIB-4 has relatively high diagnostic value for detecting liver fibrosis in patients with hepatitis B when the diagnostic threshold value was more than 2.0. We expect further studies to confirm our analysis.

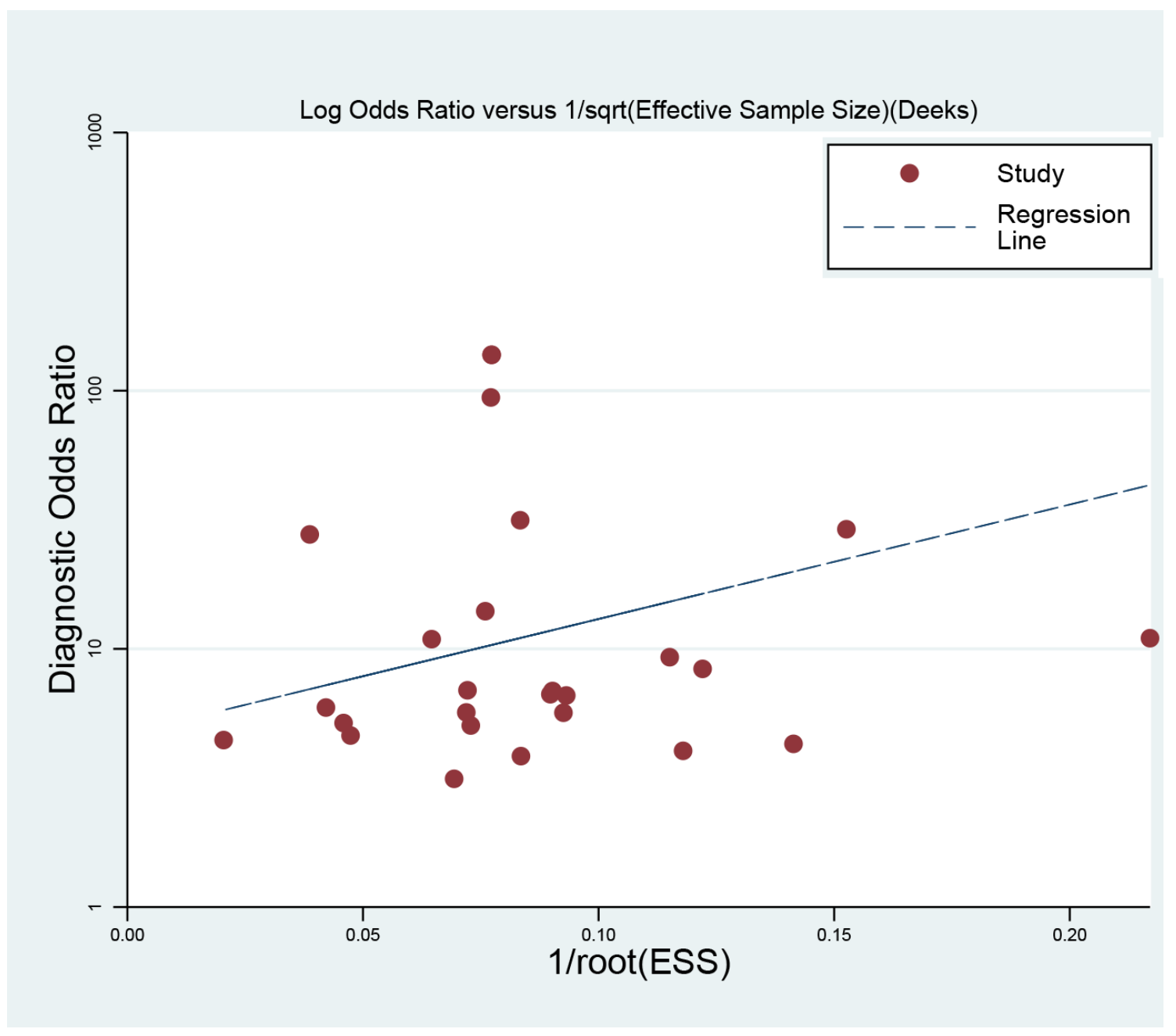

Figure 6: Deek's funnel plot to evaluate the publication bias. 


\section{MATERIALS AND METHODS}

\section{Literature search}

A systematic search was conducted for relevant articles published in the PubMed, Embase, Web of Science, and Chinese National Knowledge Infrastructure from inception to October 31, 2016. The following keywords are used: FIB-4, aspartate aminotransferase, AST, alanine aminotransferase, ALT, platelet, PLT, hepatitis B, liver fibrosis, and cirrhosis. The language was restricted in Chinese and English. We also retrieve the reference lists of relevant reviews to identify to additional studies.

\section{Selection criteria}

The included studies must meet the following criteria: (1) all the patients with liver fibrosis must be diagnosed through the gold standard (liver biopsy). (2) studies provided diagnostic value of FIB-4 for liver fibrosis. (3) study must present sufficient data to allow calculation of the diagnostic value: True positive (TP), false positive (FP), false negative (FN), and true negative (TN). Duplicate publications, studies without qualified data, focused on other diseases, and letters, reviews, case reports and editorials were excluded.

\section{Data extraction}

For each study included in the meta-analysis, the following information was extracted: the first author, publication year, region, mean age, sample size, study design, study population, length of tissue, cut-off value of diagnostic, four data (TP, FP, FN, TN). Two authors (ZJ and LQX) independently extract this information by using a standard excel sheet, and cross check the data. Any disputes were solved by the third investigator (CLZ).

\section{Quality evaluation}

We used the Quality Assessment of Diagnostic Accuracy Studies 2 (QUADAS-2) to assess the quality of included studies. We used a quantitative method to assess the studies. The QUADAS-2 included 14 items [35]. Each key domain includes two sections: risk of bias and applicability. If answers to all signaling questions for a domain are 'yes', then we could judge the risk of bias is low. If any question is answered 'no', potential bias exists. Concerns about applicability are judged as 'low', 'high', or 'unclear'. We defined 'Yes' as one scores.

\section{Statistical analysis}

We used the Stata 14 software (StataCorp LP, College Station, TX, USA) to perform all statistical analyses. The heterogeneity within studies was evaluated by Q Test and $I^{2}$ test, and $I^{2}>50 \%$ presented the existence of heterogeneity [36]. The bivariate regression model was used to calculate the polled sensitivity, specificity, positive and negative likelihood ratios (PLRs and NLRs), diagnostic odds ratio (DOR) and their $95 \%$ confidence intervals (CIs) [37]. We also calculated the area under the receiver operator characteristic curve (SROC, AUC). The AUC ranged from 0 to 1 , and an AUC of 1 represents the perfect discrimination ability, while an $\mathrm{AUC}<0.5$ shows a poor diagnostic ability [38]. We also conducted a subgroup based on cut-off value. We used the Deek's funnel plot to assess the publication bias, and Fagan plots shows the relationship between the prior probability, the likelihood ration, and posterior test probability [39]. $P<0.05$ was considered to be significant.

\section{Abbreviations}

Aspartate aminotransferase to platelet ratio index TP, APRI; Fibrosis index based on the 4 factor, FIB-4; aspartate aminotransferase, AST; alanine aminotransferase, ALT; platelet, PLT; Quality Assessment of Diagnostic Accuracy Studies 2, QUADAS-2; true positives; FP, false positives; FN, false negatives; TN, true negatives; ROC, Receiver Operating Characteristic; SROC, summary receiver operator characteristic PLRs, positive likelihood ratios; NLRs, negative likelihood ratios; DORs, diagnostic odds ratios; CI, confidence intervals.

\section{ACKNOWLEDGMENTS}

$\mathrm{YZ}$ and CLZ designed this study and contributed substantially to the design of the search strategy. $\mathrm{YZ}$ and CLZ searched and selected the trials and extracted data. $\mathrm{ZJ}$ and $\mathrm{YZ}$ performed the analysis and interpreted the data. YZ wrote the manuscript. YZ, LQX and CLZ critically reviewed the manuscript. YZ, ZJ and CLZ participated in the data extraction and critically revised it. YZ and CLZ proofread the final version. All authors read and approved the final manuscript.

\section{CONFLICTS OF INTEREST}

The authors declare that they have no conflict of interest.

\section{REFERENCES}

1. Ganem D and Prince AM. Hepatitis B virus infection-natural history and clinical consequences. N Engl J Med. 2004; 350:1118-1129.

2. Fattovich G, Bortolotti F and Donato F. Natural history of chronic hepatitis B: special emphasis on disease progression and prognostic factors. J Hepatol. 2008; 48:335-352. 
3. Basar O, Yimaz B, Ekiz F, Ginis Z, Altinbas A, Aktas B, Tuna Y, Coban S, Delibas N and Yuksel O. Non-invasive tests in prediction of liver fibrosis in chronic hepatitis B and comparison with post-antiviral treatment results. Clin Res Hepatol Gastroenterol. 2013; 37:152-158.

4. Sterling RK, Lissen E, Clumeck N, Sola R, Correa MC, Montaner J, S SM, Torriani FJ, Dieterich DT, Thomas DL, Messinger D and Nelson M. Development of a simple noninvasive index to predict significant fibrosis in patients with HIV/HCV coinfection. Hepatology. 2006; 43:1317-1325.

5. Ucar F, Sezer S, Ginis Z, Ozturk G, Albayrak A, Basar O, Ekiz F, Coban S, Yuksel O, Armutcu F and Akbal E. APRI, the FIB-4 score, and Forn's index have noninvasive diagnostic value for liver fibrosis in patients with chronic hepatitis B. Eur J Gastroenterol Hepatol. 2013; 25:1076-1081.

6. Bonnard P, Sombie R, Lescure FX, Bougouma A, GuiardSchmid JB, Poynard T, Cales P, Housset C, Callard P, Le Pendeven C, Drabo J, Carrat F and Pialoux G. Comparison of elastography, serum marker scores, and histology for the assessment of liver fibrosis in hepatitis B virus (HBV)infected patients in Burkina Faso. AM J Trop Med Hyg. 2010; 82:454-458.

7. Chen B, Ye B, Zhang J, Ying L and Chen Y. RDW to platelet ratio: a novel noninvasive index for predicting hepatic fibrosis and cirrhosis in chronic hepatitis B. PLoS One. 2013; 8:e68780.

8. Erdogan S, Dogan HO, Sezer S, Uysal S, Ozhamam E, Kayacetin $\mathrm{S}$ and Koca $\mathrm{Y}$. The diagnostic value of non-invasive tests for the evaluation of liver fibrosis in chronic hepatitis B patients. Scand J Clin Lab Invest. 2013; 73:300-308.

9. Ji D, Shao Q, Han P, Zhang J, Niu X, Li B, Li F and Chen G. Analysis of the efficiency of Fibroscan combined with serologic markers for diagnosing liver fibrosis. Med J Chin PLA. 2011:1136-1138.

10. Jing L, Ying-xia L, Chang-feng D, Si-min Y, Sha-xi LI, Jing Y, Chuan-tie C, Mei-fen Z, Yi-min L and Zhong-tian P. ARFI, Forns index, FIB-4 and APRI diagnosis liver ifbrosis in patients with chronic liver diseases. Chinese Journal of Liver Diseases. 2014:18-21.

11. Kim BK, Kim DY, Park JY, Ahn SH, Chon CY, Kim JK, Paik YH, Lee KS, Park YN and Han KH. Validation of FIB-4 and comparison with other simple noninvasive indices for predicting liver fibrosis and cirrhosis in hepatitis B virus-infected patients. Liver Int. 2010; 30:546-553.

12. Koksal AR, Alkim H, Boga S, Ergun M, Bayram M, Ozguven BY and Alkim C. Effect of Entecavir and Tenofovir Treatment on Noninvasive Fibrosis Scores: Which One Is Better? Am J Ther. 2016; 23: e429-e438.

13. Li J, Gordon SC, Rupp LB, Zhang T, Boscarino JA, Vijayadeva V, Schmidt MA and Lu M. The validity of serum markers for fibrosis staging in chronic hepatitis B and C. J Viral Hepat. 2014; 21:930-937.
14. Li X, Zhang Z, Cai Q, Xie Q, Zhang Y, Jiang X, Li $X$ and Yin $H$. Judgement of liver fibrosis by gamaglutamyltransferase to qHBsAg ratio in patients with chronic hepatitis B. J Prac Hepatol. 2015; 18:141-144.

15. Liu HB, Zhou JP, Zhang Y, Lv XH and Wang W. Prediction on liver fibrosis using different APRI thresholds when patient age is a categorical marker in patients with chronic hepatitis B. Clin Chim Acta. 2011; 412:33-37.

16. Liu XD, Wu JL, Liang J, Zhang T and Sheng QS. Globulinplatelet model predicts minimal fibrosis and cirrhosis in chronic hepatitis B virus infected patients. World J Gastroenterol. 2012; 18:2784-2792.

17. Mallet V, Dhalluin-Venier V, Roussin C, Bourliere M, Pettinelli ME, Giry C, Vallet-Pichard A, Fontaine H and Pol S. The accuracy of the FIB-4 index for the diagnosis of mild fibrosis in chronic hepatitis B. Aliment Pharmacol Ther. 2009; 29:409-415.

18. Sebastiani G, Castera L, Halfon P, Pol S, Mangia A, Di Marco V, Pirisi M, Voiculescu M, Bourliere M and Alberti A. The impact of liver disease aetiology and the stages of hepatic fibrosis on the performance of non-invasive fibrosis biomarkers: an international study of 2411 cases. Aliment Pharmacol Ther. 2011; 34:1202-1216.

19. Seto WK, Lee CF, Lai CL, Ip PP, Fong DY, Fung J, Wong DK and Yuen MF. A new model using routinely available clinical parameters to predict significant liver fibrosis in chronic hepatitis B. PLoS One. 2011; 6:e23077.

20. Shrivastava R, Sen S, Banerji D, Praharaj AK, Chopra GS and Gill SS. Assessment of non-invasive models for liver fibrosis in chronic hepatitis B virus related liver disease patients in resource limited settings. Indian $\mathrm{J}$ Pathol Microbiol. 2013; 56:196-199.

21. Wang H, Xue L, Yan R, Zhou Y, Wang MS, Cheng MJ and Huang HJ. Comparison of FIB-4 and APRI in Chinese HBV-infected patients with persistently normal ALT and mildly elevated ALT. J Viral Hepat. 2013; 20: e3-e10.

22. Wang Y, Xu MY, Zheng RD, Xian JC, Xu HT, Shi JP, Li SB, Qu Y, Dong YW and Lu LG. Prediction of significant fibrosis and cirrhosis in hepatitis $\mathrm{B}$ e-antigen negative patients with chronic hepatitis B using routine parameters. Hepatol Res. 2013; 43:441-451.

23. Wu SD, Ni YJ, Liu LL, Li H, Lu LG and Wang JY. Establishment and validation of a simple noninvasive model to predict significant liver fibrosis in patients with chronic hepatitis B. Hepatol Int. 2012; 6:360-368.

24. Wu SD, Wang JY and Li L. Staging of liver fibrosis in chronic hepatitis B patients with a composite predictive model: a comparative study. World J Gastroenterol. 2010; 16:501-507.

25. Xu WS, Qiu XM, Ou QS, Liu C, Lin JP, Chen HJ, Lin S, Wang WH, Lin SR and Chen J. Red blood cell distribution width levels correlate with liver fibrosis and inflammation: a noninvasive serum marker panel to predict the severity of fibrosis and inflammation in patients with hepatitis B. Medicine (Baltimore). 2015; 94:e612. 
26. Xun YH, Zang GQ, Guo JC, Yu XL, Liu H, Xiang J, Liu J and Shi JP. Serum hepatitis B surface antigen quantification as a useful assessment for significant fibrosis in hepatitis B e antigen-positive hepatitis B virus carriers. J Gastroenterol Hepatol. 2013; 28:1746-1755.

27. Zeng DW, Liu YR, Zhang JM, Zhu YY, Lin S, You J, Li YB, Chen J, Zheng Q, Jiang JJ and Dong J. Serum ceruloplasmin levels correlate negatively with liver fibrosis in males with chronic hepatitis B: a new noninvasive model for predicting liver fibrosis in HBV-related liver disease. PLoS One. 2013; 8:e77942.

28. Zhu CL, Li WT, Li Y and Gao RT. Serum levels of tissue inhibitor of metalloproteinase-1 are correlated with liver fibrosis in patients with chronic hepatitis B. J Dig Dis. 2012; 13:558-563.

29. Zhu X, Wang LC, Chen EQ, Chen XB, Chen LY, Liu L, Lei XZ, Liu C and Tang H. Prospective evaluation of FibroScan for the diagnosis of hepatic fibrosis compared with liver biopsy/AST platelet ratio index and FIB-4 in patients with chronic HBV infection. Dig Dis Sci. 2011; 56:2742-2749.

30. Li ZZ, Shen LF, Li YY, Chen P and Chen LZ. Clinical utility of microRNA-378 as early diagnostic biomarker of human cancers: a meta-analysis of diagnostic test. Oncotarget. 2016; 7:58569-58578. doi: 10.18632/oncotarget.10707.

31. Jin W, Lin Z, Xin Y, Jiang X, Dong Q and Xuan S. Diagnostic accuracy of the aspartate aminotransferase-toplatelet ratio index for the prediction of hepatitis B-related fibrosis: a leading meta-analysis. BMC Gastroenterol. 2012; $12: 14$.
32. Salkic NN, Jovanovic P, Hauser G and Brcic M. FibroTest/ Fibrosure for significant liver fibrosis and cirrhosis in chronic hepatitis B: a meta-analysis. Am J Gastroenterol. 2014; 109:796-809.

33. Chou R and Wasson N. Blood tests to diagnose fibrosis or cirrhosis in patients with chronic hepatitis $\mathrm{C}$ virus infection. Ann Intern Med. 2013; 159:372.

34. Moher D, Liberati A, Tetzlaff J and Altman DG. Preferred reporting items for systematic reviews and meta-analyses: the PRISMA statement. Int J Surg. 2010; 8:336-341.

35. Whiting PF, Rutjes AW, Westwood ME, Mallett S, Deeks JJ, Reitsma JB, Leeflang MM, Sterne JA and Bossuyt PM. QUADAS-2: a revised tool for the quality assessment of diagnostic accuracy studies. Ann Intern Med. 2011; 155:529-536.

36. Higgins JP, Thompson SG, Deeks JJ and Altman DG. Measuring inconsistency in meta-analyses. BMJ. 2003; 327:557-560.

37. Reitsma JB, Glas AS, Rutjes AW, Scholten RJ, Bossuyt $\mathrm{PM}$ and Zwinderman AH. Bivariate analysis of sensitivity and specificity produces informative summary measures in diagnostic reviews. J Clin Epidemiol. 2005; 58:982-990.

38. Hamza TH, Arends LR, van Houwelingen HC and Stijnen T. Multivariate random effects meta-analysis of diagnostic tests with multiple thresholds. BMC Mrd Res Methodol. 2009; 9:73.

39. Song F, Khan KS, Dinnes J and Sutton AJ. Asymmetric funnel plots and publication bias in meta-analyses of diagnostic accuracy. Int J Epidemiol. 2002; 31:88-95. 